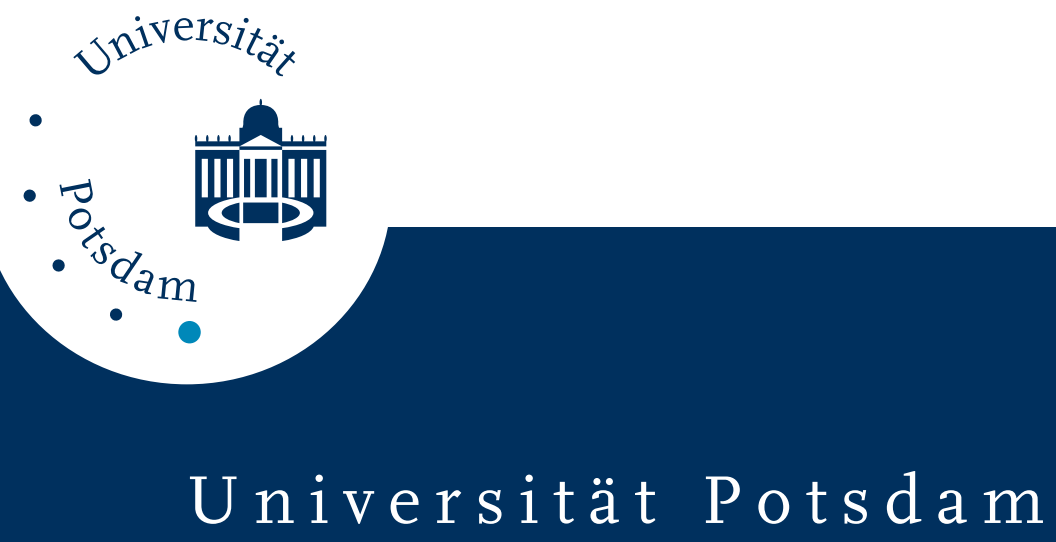

M. B. Biddle, S. E. Rickert, J. B. Lando, André Laschewsky

\title{
The use of the Langmuir-Blodgett technique to obtain ultra-thin polar films
}

first published in:

Sensors and Actuators, 20 (1989) S. 307-313, ISSN 0250-6874, DOI $10.1016 / 0250-6874(89) 80130-1$

Postprint published at the Institutional Repository of Potsdam University:

In: Postprints der Universität Potsdam

Mathematisch-Naturwissenschaftliche Reihe ; 98

http://opus.kobv.de/ubp/volltexte/2009/1718/

http://nbn-resolving.de/urn:nbn:de:kobv:517-opus-17185

Postprints der Universität Potsdam

Mathematisch-Naturwissenschaftliche Reihe ; 98 


\title{
The Use of the Langmuir-Blodgett Technique to Obtain Ultra-Thin Polar Films
}

\author{
M. B. BIDDLE*, S. E. RICKERT, J. B. LANDO and A. LASCHEWSKY** \\ Department of Macromolecular Science, Case Western Reserve University, Cleveland, OH 44106 (U.S.A.)
}

(Received October 7, 1987; in revised form October 6, 1988 and May 16, 1989; accepted May 17, 1989)

\begin{abstract}
The piezoelectric and pyroelectric properties of oriented films possessing dipole moments are increasingly being used in pressure, acoustic, thermal and optical devices. The performance of these devices in many applications may be enhanced by thin-film technology. The developing LangmuirBlodgett thin-film deposition technique offers the opportunity to obtain highly oriented and uniform organic-based films in the $10-5000 \mathrm{~nm}$ thickness range. Special techniques must be used, however, to assemble these molecules in such a way as to result in polar multilayer films. Several possible deposition techniques are investigated, with one resulting in a polar and pyroelectric film about $50 \mathrm{~nm}$ thick.
\end{abstract}

\section{Introduction}

In the 1930s Irving Langmuir and Katherine Blodgett collaborated to develop a method of depositing monomolecular layers onto solid substrates $[1,2]$. In the 'classical' technique, an appropriate amphiphilic molecule (one possessing a hydrophobic tail and a hydophilic head) is deposited onto a water surface, usually dropwise, from a volatile carrier solvent. It may spread to form a monomolecular layer with its polar head group associated with the water. The layer is then compressed to a two dimensional quasi-solid, one molecule thick, by sliding a hydrophobic barrier across the very top of the surface. If a suitable substrate is passed in a vertical direction through the water surface while the surface pressure is maintained at a constant value, a monolayer can be deposited with each transverse of the airwater interface. Conditions such as type of molecule, temperature, aqueous phase $\mathrm{pH}$ and dipping speed are very important deposition

*Present address: Dow Chemical, 2800 Mitchell Drive, Walnut Creek, CA 94598, U.S.A.

**Present address: Universität Mainz, Institut für Organische Chemie, J. J. Becher-Weg 18-20, D-6500 Mainz, F.R.G parameters. These parameters are discussed in numerous references (for example, see refs. 3-8).

As is evident from the above and many other recent sources, the Langmuir-Blodgett (LB) filmdeposition technology is experiencing a significant resurgence of interest. The $\mathrm{LB}$ technique offers a means to deposit a highly oriented film of very controlled thickness. The 'thinness' can obviously range from the molecular length upwards, depending merely on the number of layers and the length(s) of compounds to be deposited. The nature of the film can be altered very easily (within limits) simply by altering the molecule to be deposited. This type of 'molecular engineering" is very attractive to many technological and research areas, including electronics. A number of researchers have specifically investigated the dipole effect in LB films already, with several mentioning piezo- and pyroelectric possibilities [4,9-15, 27]. British and Russian groups have demonstrated piezoelectric and pyroelectric properties in several types of LB multilayers [16-20].

Films can be deposited in several modes, depending on the substrate surface treatment and dipping methodology, as shown schematically in Fig. 1. This is a very important point for the devices in question. As is evident from the Figure, a spontaneous polarization should exist in films of polar molecules deposited in the $X$ or $Z$ mode. These deposition modes result in non-centrosymmetric multilayers. No polarization will exist with the centrosymmetric $Y$ films due to cancellation of the dipole moments by alternating layers. Unfortunately, deposition of the $Y$-type is usually obtained. The HHTT (head to head, tail to tail) organization is the most common arrangement found in the bulk crystals due to polar-polar attraction. It becomes even more likely as the head groups become more polar. There are, however, numerous possible techniques to obtain polar films from materials that normally deposit in a $Y$-type manner.

We focused on two basic approaches to obtaining polar multilayer films. The first was to try to force the molecules to assemble in an $X$ or $Z$ manner through special deposition procedures 

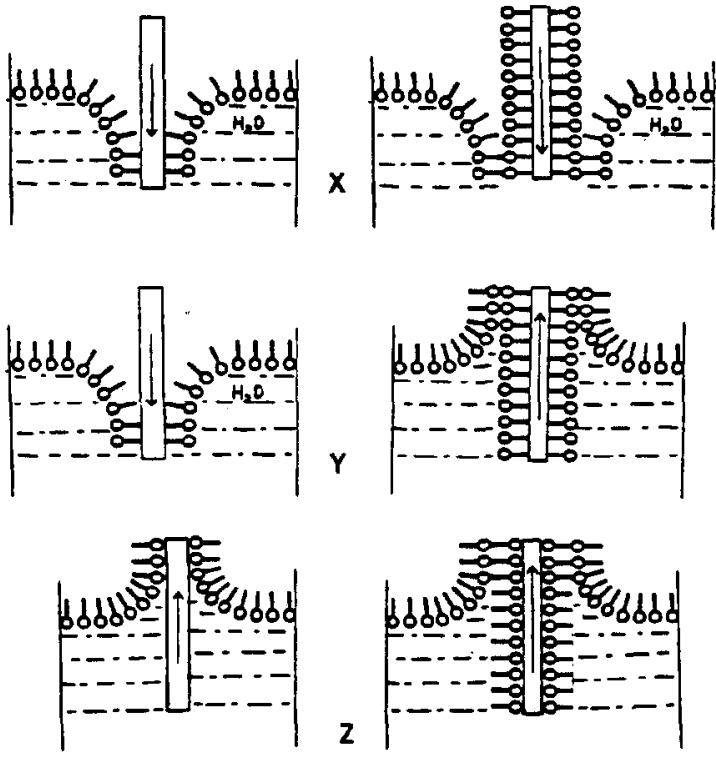

Fig. 1. Nomenclature for different deposition types.

coupled with polymerizations. The polymerizations were employed to discourage the type of 'molecular rearrangement' suggested by previous researchers [21-26]. This approach has previously proved to be successful with vinyl stearate [24].

The second approach makes use of the natural desire of the molecules to associate polar head groups in adjacent layers. The alternating layer technique constructs multilayers from two different materials, one material possessing a much larger dipole than the other. The materials are deposited alternately with their polar head groups adjacent to one another. Even though the dipoles may oppose one another, a net dipole remains, since one is much larger than the other. If the correct materials are chosen, the dipoles may even face in the same direction in the multilayer [19]. Alternating approaches have been demonstrated by British groups $[16,19,20]$. Special 'double-dip' troughs have been fabricated to facilitate this technique $[24,28-30]$.

Films were deposited in $X, Z$ and horizontal modes using a single polymerizable material. Alternating layers were also manufactured using two different combinations of materials. Glancing angle X-ray diffraction was used to determine the organization (HHTT or HTHT) of the molecules. The type of organization for single-material multilayers determines whether or not the particular assembly is non-centrosymmetric. Ultimately, electrical measurements were made to determine if any of the structures were polar and pyroelectric

\section{Experimental}

\subsection{Substrates}

Quartz slides $(5 \times 1 \times 0.15 \mathrm{~cm})$ were used for the polymerization studies requiring UV-transparent substrates. Glass slides $(12 \times 2.5 \times 0.15 \mathrm{~cm})$ and $2.5 \mathrm{~cm}$ diameter silicon wafers were used for the X-ray studies. All substrates were cleaned using a standard RCA degreasing technique consisting of consecutive five minute ultrasonic immersions in trichloroethylene, acetone and then methanol [31]. This degreasing step was performed before deposition took place on any substrates.

In certain cases, hydrophobic substrates were needed. The substrates were suspended above hexamethyldisilazane (HMDS) liquid. The pure vapor reacted with the oxide surface, attaching methyl groups that formed a reasonably hydrophobic surface [32]. Contact angles went from $10-15^{\circ}$ for the freshly cleaned substrates to over $70^{\circ}$ after treatment.

Metal-insulator-metal (MIM) structures were made for the electrical studies. Aluminum electrodes were evaporated through contact masks onto cleaned glass slides or silicon wafers, then the substrates were re-cleaned. After film deposition, top aluminum electrodes were then evaporated through shadow masks at a slow rate of about $1 \mathrm{~nm} \mathrm{~s}^{-1}$ to minimize any degradation of the LB films. Some studies were also carried out on silicon wafers with electrodes patterned via photolithography techniques. In this case, the LB film was relied upon to protect the portions of exposed bottom aluminum during etching of the top electrode pattern. The aluminum etching solution was used at a relatively low temperature of $30^{\circ} \mathrm{C}$ to minimize damage to the LB films.

\subsection{Amphiphilic Materials}

Most of the special depositions that were attempted required a polymerizable material. 10,12 nonacosadiynoic acid (also known as 16-8 diacetylene) was used because of its ease of polymerization upon UV exposure. The reaction of the diacetylene units is fast and can be followed rather easily with UV spectroscopy.

A Perkin-Elmer Lamda $5 \mathrm{UV} /$ Vis spectrophotometer was used to evaluate the polymerization conditions for the $X, Z$ and horizontal deposition techniques. For all three cases, material was deposited onto quartz slides. The $X$ deposition study used samples that were exposed to UV radiation underwater for various periods of time. The samples for the $Z$ deposition study were exposed in a nitrogen-purged polymerization chamber. Finally, the horizontal deposition study was performed on material that was first polymerized at the water surface before deposition. 
A molecule with a potentially higher dipole moment was desired for the alternating layer study. Laschewsky synthesized 4-octadecyloxy aniline with this type of application in mind. It was alternated with both diacetylene and 22-tricosenoic acid, a well-known LB material with excellent film-forming properties.

\subsection{Film Deposition}

Lauda troughs were used to contain and manipulate the monolayers at the water surface. Stepper motors were used to drive gear boxes for the dipping of the substrates through the monolayers. A slow dipping speed of $3 \mathrm{~mm} \mathrm{~min}^{-1}$ was used in most cases. Dipping was controlled and data collected through interfaces with IBM personal computers. The software was developed in our laboratory by Shutt [33].

The water for the subphase was purified sequentially via filtration, reverse osmosis and distillation to provide water with a resistance of $15-18$ Mohm. In some cases $\mathrm{CdCl}_{2}$ was added to the subphase at a concentration of about $10^{-4} \mathrm{M}$ in order to form the Cd salt of the acid. This allowed $\mathrm{X}$-ray diffraction to take place from a fewer number of layers, typically $7-8$ rather than the $15-20$ needed for good diffraction from the free acid.

Unpolymerized diacetylene was maintained at a surface pressure of $20 \mathrm{mN} \mathrm{m}^{-1}$ and the subphase was kept at $15^{\circ} \mathrm{C}$ unless stated otherwise. Diacetylene polymerized at the water surface before deposition was maintained at $25 \mathrm{mN} \mathrm{m}^{-1}$ and $20^{\circ} \mathrm{C}$. The 22-tricosenoic acid was deposited at $27 \mathrm{mN} \mathrm{m}^{-1}$ and $20^{\circ} \mathrm{C}$, while the oxyaniline material was deposited at about $20 \mathrm{mN} \mathrm{m}^{-1}$ and $20^{\circ} \mathrm{C}$.

\section{4. $X, Z$ and Horizontal Depositions}

As discussed earlier, most materials will deposit in a centrosymmetric HHTT fashion. In order to force the molecules to assemble in a noncentrosymmetric HTHT structure, procedures such as allowing monolayers to deposit only on uptrips or downtrips by removing the material between steps were attempted. Polymerization of each freshly deposited monolayer was effected to discourage molecular rearrangement and film loss during passage through the clean water surface.

Hydrophobic glass or silicon substrates were dipped through spread monolayers of diacetylene as the first step in $X$-type deposition. A monolayer was deposited on the downtrip and the substrate was left underwater. The remaining material on the water surface was then removed via aspiration. A UV pencil lamp, fitted in a quartz tube, was submerged in front of the substrate. The freshly deposited monolayer was then exposed to radiation for a period of time determined by the
UV polymerization study. After the exposure, the substrate was raised through the clean water surface. The water surface was cleaned further, then another monolayer spread and the process repeated until the desired number of layers were deposited. $Z$-type films were prepared in a similar manner, except that hydrophilic substrates were used and polymerizatons of each layer were carried out in a nitrogen atmosphere rather than underwater.

Two types of depositions were attempted using diacetylene pre-polymerized at the water surface. Diacetylene was spread on the water surface and maintained at a surface pressure of $10 \mathrm{mN} \mathrm{m}^{-1}$. The area above the monolayer was then purged with nitrogen before a UV lamp housed in the trough top was activated. The polymerized material was then compressed to $25 \mathrm{mN} \mathrm{m}^{-1}$ before deposition.

$Z$-type deposition with this pre-polymer material was effected by merely reducing the pressure to about $10 \mathrm{mN} \mathrm{m}^{-1}$ during downtrips. No deposition took place during the low-pressure downtrip, as determined by the lack of barrier movement. When deposition is occurring, the compression barrier will move in, reducing the monolayer area in order to maintain pressure as material is removed from the surface. This processing technique saved considerable time and effort.

Horizontal deposition was effected by placing the substrates parallel with the water/monolayer surface. A hole is 'punched' in the polymerized diacetylene as the large surface penetrates the interface. The integrity of the monolayer was substantial, as a depression of about $1 \mathrm{~cm}$ below the surface was possible before the film broke and water rushed over the sample. The barrier moved by an amount corresponding to the area of the substrate, suggesting that a uniform monolayer had indeed been deposited.

\subsection{Alternating Layers}

Alternating layers were constructive in our laboratory without the use of an automated double-dip trough. Two troughs were used; one containing either the 22-tricosenoic acid or the diacetylene acid and the other containing the oxyaniline. The acids were typically deposited on the downtrips and the oxyaniline on the uptrips. Since two separate troughs were used, deposition was prohibited on the uptrips for the acids and on the downtrips for the oxyaniline by lowering the pressure, as was done for the pre-polymerized diacetylene. However, care had to be taken not to lower the pressure too far or the previously deposited material would re-deposit onto the water surface. 


\subsection{Characterization}

Glancing angle $\mathrm{X}$-ray diffraction was performed on the various samples with a Philips diffractometer. The diffractometer used $\mathrm{Cu} \mathrm{K} \alpha$ radiation with a wavelength of $0.1542 \mathrm{~nm}$. The glancing angle diffraction results will give the repeat distance for the LB films provided that uniform multilayers have been deposited. A HHTT structure will result in a repeat distance of roughly two molecular lengths, while a HTHT structure will have a repeat distance roughly equal to one molecular length.

Electrical characterization was carried out on a Wentworth Labs Probing Station. Tungsten probes were used to make contact to the aluminum pads in most cases. However, a gold wire attached to a tungsten probe proved to be less damaging to the top electrodes. The top aluminum was evaporated onto the soap-like multilayers and therefore exhibited poorer adhesion than the bottom metallization deposited directly on to the glass and silicon substrates. For the larger area electrodes used in the pyroelectric measurements, gold wires were bonded to the aluminum using conductive epoxy.

Current versus voltage $(I V)$ measurements were made using an EG \& G Princeton Applied Research model $410 C-V$ Plotter with the oscillator turned off to function as a programmable voltage source. A Keithley 616 Digital Electrometer functioned as the current meter. The samples were scanned at a rate of $100 \mathrm{mV} \mathrm{s}^{-1}$. No differences were found for the tungsten and the gold wire top electrodes in these tests. The electrometer was also used to measure charge accumulation and depletion for the pyroelectric studies.

\section{Results and Discussion}

\section{1. $X$-ray Diffraction}

Samples produced by either the $X$ or $Z$ deposition techniques resulted in multilayers with layer spacings corresponding to HHTT structures. The indicated $d$-spacings were consistently about $6.4 \mathrm{~nm}$ for the Cd-salt samples and about $5.4 \mathrm{~nm}$ for the free acid samples. Since the theoretical molecular length of our diacetylene is about $3.5 \mathrm{~nm}$, this can only correspond to a bilayer repeat. The less than theoretical repeat distance is common for LB films, and is typically explained by molecular tilting [34,35]. An interdigitation mechanism has also been proposed [36].

Since the first two techniques resulted only in HHTT multilayers, it was felt that the molecules must be reorganizing during deposition. Unlike vinyl stearate, the more polar diacetylene acid molecules still find a way to associate head

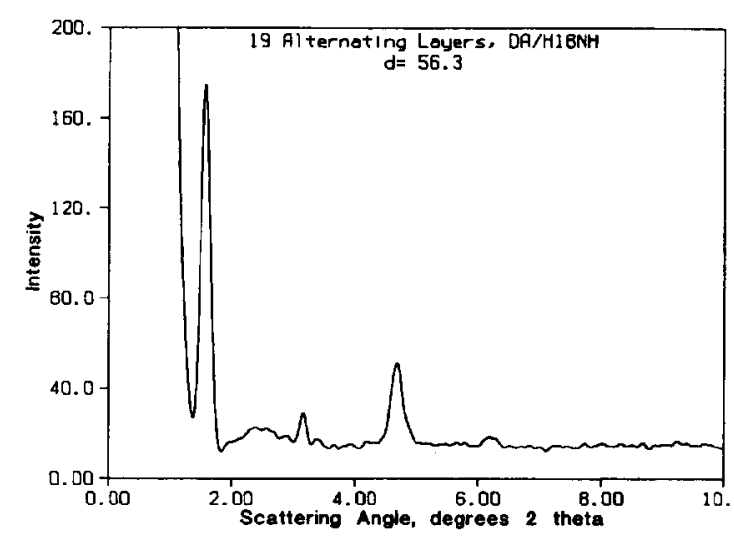

Fig. 2. Glancing angle $X$-ray diffraction spectrum for an alternating film of 19 layers.

groups, even with the unique deposition procedures. To 'lock' the depositing molecules in place, diacetylene was pre-polymerized at the water surface before deposition. Both $Z$ and horizontal depositions, however, resulted in only HHTT indicated molecular structures when examined with glancing angle $\mathrm{X}$-ray diffraction.

One way to work with this extreme propensity for the polar head groups to associate is to use the alternating layer technique described earlier. An $X$-ray diffraction spectrum from a 19-layer sample of diacetylene alternated with the oxyaniline is shown in Fig. 2. The calculated layer spacing is about $5.6 \mathrm{~nm}$, indicating that the molecules are indeed arranged in the usual HHTT organization.

The $d$-spacing calculated from the X-ray diffraction of a 69-layer sample of pure oxyaniline was $5.7 \mathrm{~nm}$. This implies that the aniline molecule is arranged HHTT and is essentially perpendicular to the substrate, since the estimated molecular length is $2.86 \mathrm{~nm}$. A slight amount of tilting is probably present, since this estimate does not allow for bilayer intermolecular spacing. If we use the 2.86 length for the aniline and the $2.7 \mathrm{~nm}$ length for the 16-8 diacetylene (5.4/2), we get an estimated $5.56 \mathrm{~nm}$ bilayer repeat. This is essentially equal to the $5.6 \mathrm{~nm}$ observed spacing for the alternating structure. Furthermore, the intensity is comparable to that of straight diacetylene systems, suggesting that a well-organized multilayer structure has been obtained.

\subsection{Electrical Characterization}

Several types of electrical tests were performed on the MIM structures. Current versus voltage (IV) measurements were made to verify that the multilayers were continuous (good insulators). A voltage ramp of $100 \mathrm{mV} \mathrm{s}^{-1}$ was applied starting from $0 \mathrm{~V}$. The resistance of these films in the linear 
range of the $I V$ curves was in the Gohm range or greater. At higher voltages, the current begins to deviate from linearity. This deviation becomes precipitous as the insulator begins to suffer 'softbreakdown'. Full breakdown of the insulator does not occur at these small currents and the curves can be reproduced. At currents on the order of $10^{-7} \mathrm{~A}$, however, the insulator does suffer permanent breakdown (manifested as a complete short). The current densities that are withstood by these devices are of the order of $10^{-8} \mathrm{~A} \mathrm{~cm}^{-2}$.

In order to determine if a polar structure had been produced, a pyroelectric measurement was made on an alternating-layer sample using an approach similar to that demonstrated by Smith et al. [16]. In this case, the sample was placed on the heating stage of the probing station. The entire station was enclosed in an aluminum box to reduce air currents and electronic interference. Gold wires were attached to the electrodes via a room-temperature cure silver conductive epoxy to facilitate making electrical contact.

The electrometer was operated in Coulomb mode in order to evaluate the change in charge with temperature. The stage was heated electrically to $20^{\circ} \mathrm{C}$, then cooled with circulating water to $10^{\circ} \mathrm{C}$. This was repeated several times. The $10^{\circ}$ temperature change produced an average decrease in charge accumulation of $2.75 \times 10^{-10} \mathrm{C} \mathrm{cm}^{-2}$ upon heating and an average increase of $4.0 \times 10^{-10} \mathrm{C} \mathrm{cm}^{-2}$ upon cooling. This cycle was very reproducible.

If this alternating multilayer is non-centrosymmetric as expected, it should contain a spontaneous polarization, $P_{\mathrm{s}}$. If $\boldsymbol{P}_{\mathrm{s}}$ is a function of temperature, we will have a pyroelectric effect. The pyroelectric coefficient is defined as:

$p=\mathrm{d} P_{\mathrm{s}} / \mathrm{d} T$

where $p=$ pyroelectric coefficient, $\mathrm{C} \mathrm{cm}^{-2} \mathrm{~K}^{-1}$ and $T=$ temperature change, $K$.

Since we cannot easily measure $\boldsymbol{P}_{\mathrm{s}}$, we measure the charge that develops on the surfaces perpendicular to the polar axis. The LB film is a dielectric and the compensation conduction charges are too slow to follow the induced change in $P_{s}$. A pyroelectric coefficient can be calculated from

$Q=p \Delta T$

where $Q=$ charge in Coulombs and $T=$ temperature change, $K$.

Averaging the two changes for heating and cooling (as Smith et al. did), gives $3.4 \times$ $10^{-10} \mathrm{C} \mathrm{cm}^{-2}$ for a $10^{\circ} \mathrm{C}$ change. Using this number, the calculated value for $p$ comes out to $3.4 \times 10^{-11} \mathrm{C} \mathrm{cm}^{-2} \mathrm{~K}^{-1}$, which is of the same order of magnitude as for the saturated acid/ amine systems tested by Smith et al. [16]. However, a 22-tricosenoic/amine system evaluated by Jones $e t$ al. [19] exhibited a coefficient about six times greater than the one we measured. It was hoped that the oxyaniline system would be better than the amine systems due to a higher suspected dipole moment. With more controlled deposition, a more uniform alternating structure could be produced, with possibly higher pyroelectric response. This is still one to two orders of magnitude less than coefficients reported for PVDF [37], although these ultra-thin films should prove to be more sensitive due to their lower thermal mass.

One of the reasons for the relatively low coefficient lies in the fact that the dipole moment is concentrated at the ends of the molecules. The non-polar 'tails' of the molecules act to dilute the effect on a volume basis. The effect may also be one of secondary pyroelectricity only; i.e., simply due to the change in charge/volume from the thermal expansion and contraction. Primary pyroelectric activity requires molecular dipole moment changes.

To help understand the 'rigidity' of this system, FT-IR was used to determine if salt formation occurred between the aniline and acid groups. Separate LB films of the oxyaniline, the tricosenoic acid and an alternating structure using these two materials were produced on germanium plates for transmission IR spectroscopy. The results are shown in Fig. 3. Salt formation should be accompanied by a loss of the $\mathrm{C}=\mathrm{O}$ stretching vibration at about $1700 \mathrm{~cm}^{-1}$ and the appearance of a peak or peaks between 1500 and $1550 \mathrm{~cm}^{-1}$ for the $\mathrm{NH}_{3}^{+}$and the $\mathrm{COO}^{-}$.

There is a reduction in the relative height of the $\mathrm{C}=\mathrm{O}$ peak at $1700 \mathrm{~cm}^{-1}$, but this is probably simply due to a roughly $50 \%$ dilution effect from the aniline in alternating layers. It does not

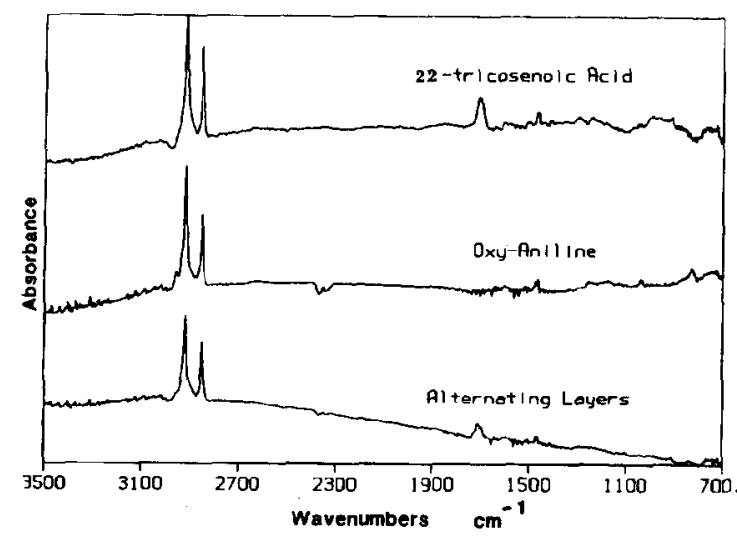

Fig. 3. FT-IR spectra of individual materials compared to an alternating layer sample. 
apear that the 'salt' $\left(-\mathrm{COO}^{-} \mathrm{H}_{3}^{+} \mathrm{N}-\right)$ ) has been formed. Smith et al. felt that they had formed the salt from their aniline/acid systems based on the changes in the IR, however, they included $10^{-3} \mathrm{M}$ ammonium sulphate in their subphase to control the $\mathrm{pH}[16]$. They could very easily have formed the salt with this component.

\section{Conclusions}

The natural propensity for these amphiphilic molecules to organize with their polar head groups associated in a centrosymmetric structure is difficult, if not impossible, to overcome using the LB technique. The alternating-layer technique represents a method to use this natural desire to form non-centrosymmetric structures. Other research groups have already demonstrated that acid/amine alternating multilayers can form films that will exhibit pyroelectric behavior. We used an acid/oxyaniline alternating system in an attempt to improve on these earlier results. Our measured pyroelectric coefficient was slightly less than that of the best system measured by previous groups using automated alternating-deposition equipment. Furthermore, our system did not appear to form the 'salt' of the acid/amine end groups as proposed by previous researchers.

\section{Acknowledgements}

We would like to thank Andrew Dewa for his great assistance in making the electrical measurements. M. B. Biddle would also like to thank Dr Gilbert W. Smith of the Royal Signals and Radar Establishment and Dr Mike C. Petty of the University of Durham, both in the U.K., for their very useful suggestions concerning the pyroelectric measurements.

\section{References}

1 K. B. Blodgett, Films built by depositing successive monomolecular layers on a solid surface, J. Am. Chem. Soc., 57 (1935) 1007.

2 K. B. Blodgett and I. Langmuir, Built-up films of barium stearate and their optical properties, Phys. Rev., $5 I$ (1937) 964.

3 G. L. Gaines, Jr., Insoluble Monolayers at Liquid-Gas Interfaces, Interscience, New York, 1966.

4 G. G. Roberts, Transducer and other applications of Lang muir-Blodgett films, Sensors and Actuators, 4 (1983) 131

5 V. K. Agarwal, Electrical behaviour of Langmuir-Blodgett films. Review, Electrocomponent Sci. Technol, 12 (1975) 1.

6 P. S. Vincett and G. G. Roberts, Electrical and photoelectrical transport properties of Langmuir-Blodgett films and a discussion of possible device applications, Thin Solid Films, 68 (1980) 135
7 V. K. Srivastava, Built-up molecular-films and their applications, in Physics of Thin Films, Vol 7, Academic Press, New York, 1973.

8 N. K. Adam, The Physics and Chemistry of Surfaces, Clarendon Press, Oxford, 1930.

9 S. K. Gupta, C. M. Singal and V. K. Srivastava, Thickness dependence of internal voltage in metal-insulator-metal structure with dissimilar electrodes, J. Appl. Phys., 48(6) (1977) 2583

10 C. M. Singal, S. K. Gupta and V. K. Srivastava, Intrinsic voltage in insulating films in aluminum-barium stearatealuminum structures, J. Appl. Phys., 49(6) (1978) 3402.

11 S. K. Gupta, A. K. Kapil, C. M. Singal and V. K Srivastava, Measurement of the work function of some metals using internal voltage in MIM structures, J. Appl. Phys., 50(4) (1979) 2852.

12 A. K. Kapil, C. M. Singal and V. K. Srivastava, Internal voltage in symmetric MIM junctions with even number of organic monolayers, J. Appl. Phys., SO(4) (1979) 2856.

13 A. K. Kapil, S. K. Gupta, C. M. Singal and V. K Srivastava, Electric dipole moment measurements by internal-voltage technique, J. Appl. Phys., SO(4) (1979) 2896.

$14 \mathrm{G}$. Marc and J. Messier, Dielectric losses in organic monomolecular layers, J. Appl. Phys., 45(7) (1974) 2832.

15 M. F. Daniel, O. C. Lettington and S. M. Small, Investigations into the Langmuir-Blodgett film formation ability of amphiphiles with cyano head groups, Thin Solid Films, 99 (1983) 61 .

16 G. W. Smith, J. W. Barton, M. F. Daniel, and N. Ratcliffe, Pyroelectric activity in non-centrosymmetric LangmuirBlodgett multilayer films, Thin Solid Films, 132 (1985) 125.

17 L. M. Blinov, N. V Dubinin, L. V. M. Khnev and S. G. Yudin, Polar Langmuir-Blodgett films, Thin Solid Films, 120 (1984) 161.

18 L. M. Blinov, N. N. Darydova, V. V. Lazarev and S. G. Yudin, Spontaneous polarization of Langmuir multimolecular films, Sov. Phys. Solid State, 24(9) (1982) 1523.

19 C. A. Jones, M. C. Petty and G. G. Roberts, Pyroelectricity in ultra-thin organic superlattices, IEEE Conf. Ferroelectrics, Lehigh, June 1986, p. 1.

20 P. Christie, C. A. Jones, M. C. Petty and G. G. Roberts, Dynamic pyroelectric response of Langmuir-Blodgett film infrared detectors, J. Phys. D: Appl. Phys., 19 (1986) L167.

21 K. Fukuda and T. Shiozawa, Conditions for formation and structural characterization of $X$-type and $Y$-type multilayers of long-chain esters, Thin Solid Films, 68 (1980) 55.

22 J. F. Stephens, Mechanisms of formation of multilayers by the Langmuir-Blodgett techniques, $J$. Colloid Interface Sci., 38(3) (1972) 557

23 A. Cemel, T. Fort, Jr., and J. B. Lando, Polymerization of vinyl stearate multilayers, J. Polym. Sci., Part A-I, 10 (1972) 2061

24 V. Enkelmann and J. B. Lando, Polymerization of ordered tail-to-tail vinyl stearate bilayers, J. Polym. Sci., Polym. Chem., 15 (1977) 1843.

25 R. C. Ehlert, Overturning of monolayers, J. Colloid Sci., 20 (1965) 387.

26 I. Langmuir, Overturning and anchoring of monolayers, Science, 87 (1938) 493.

27 P. Christie, M. C. Petty and G. G. Roberts, The preparation and dielectric properties of polybutadiene LangmuirBlodgett films, Thin Solid Films, 134 (1985) 75.

28 M. F. Daniel, J. C. Dolphin, A. J. Grant, K. E. N. Kerr and G. W. Smith, A trough for the fabrication of noncentrosymmetric Langmuir-Blodgett films, Thin Solid Films, 133 (1985) 235.

29 A. Barraud, J. Leloup, A. Gouzerh and S. Palacin, An automatic trough to make alternate layers, Thin Solid Films, 133 (1985) 117 
30 B. Holcroft, M. C Petty, G. G. Roberts and G. J. Russell, A Langmuir trough for the production of organic superlattices, Thin Solid Films, 134 (1985) 83.

31 W. Kern and D. A. Puotinen, Cleaning solutions based on hydrogen peroxide for use in silicon semiconductor technology, RCA Rev., 31(2) (1970) 187.

32 R. H. Collins and F. T. Deverse, Improvement of adhesion of photosensitive resins to silicon dioxide surfaces of semiconductors by application of hexaalkyl disilazanes for use in manufacturing field-effect transistors, IBM issue $U . S$ Patent 3549368 (July 2, 1968)

33 J. D. Shutt, Molecular multilayer thin film dielectrics in basic microelectric device structures, Master's Thesis, Case Western Reserve University, Cleveland, $\mathrm{OH}, 1986$

34 B. Tieke, G. Lieser and G. Wegner, Polymerization of diacetylenes in multilayers, J. Polym. Sci., I7(1979) 1631

35 D. Day and J. B. Lando, Morphology of crystalline diacetylene monolayers polymerized at the gas-water interface, Macromolecules, 13 (1980) 1478 and 1483 .

36 B. Belbeoch, M. Roulliay and M. Tournaire, Evidence of chain interdigitation in Langmuir-Blodgett films, Thin Solid Films, 134 (1985) 88.

37 J. Mort, Polymers as electronic materials, Adv. Phys. 29(2) (1980) 403

\section{Biographies}

Michael B. Biddle received his B.S. degree from the University of Louisville in 1978, majoring in chemical engineering. He then worked at a General Electric Plastics Applications Center, primarily in the area of composites. In 1981 he filled a position as the plastics engineer for Cummins Engine Company, for whom he continued to consult while beginning graduate studies at CWRU in 1983. Dr Biddle completed his requirements for the Ph.D. degree in the Department of Macromolecular Science in August, 1987. This work was part of his Ph.D. research. He is now working as a project leader for Dow Chemical's Western Division in Walnut Creek, CA.

Scott E. Rickert received his B.S. in chemical engineering from Cornell University in 1975 . He then attended Case Western Reserve University where he completed both his M.S. and Ph.D. in macromolecular science. After completion of his Ph.D., Dr Rickert spent one year as a research specialist in the Physics Department at the University of Pennsylvania. In 1981, he joined the faculty at Case Western Reserve University as an assistant professor in macromolecular science. From 1981-83, Dr Rickert helped to establish the Center for Adhesives, Sealants and Coatings at CWRU. In 1983, he founded and became the first director of the Polymer Microdevice Laboratory sponsored by DARPA. The PML is a facility devoted to thin-film polymer research conducted under ultra-clean conditions. Dr Rickert is recognized as one of the nation's leading experts in thin-film technology, with emphasis on the application of Langmuir-Blodgett films. He is currently on leave of absence from CWRU and is serving as president of his own company, NanoFilm Corporation.

Jerome B. Lando received his A.B. in chemistry from Cornell University, his Ph.D. from Polytechnic Institute of Brooklyn and served a postdoctoral appointment following his graduation in solid-state polymerization, also at the Polytechnic Institute of Brooklyn. His technical experience includes a position as chemist at the Research Triangle Institute in North Carolina, and a visiting professorship at the University of Mainz, F.R.G. In 1965 he was made an assistant professor of polymer science and engineering at Case Western Reserve University. In 1974 he was made a professor in that same department, and served as chairman of the Department of Macromolecular Science from 1.978 to 1985 . His research interests include organic reactions in the solid state, solid-state polymerization, pyroelectric and piezoelectric polymers as well as the electronic properties of polymers and thin films.

A. Laschewsky obtained his Ph.D. under the supervision of Dr Helmut Ringsdorf at the University of Mainz in 1986 . He then served a postdoctoral appointment at IBM and another in 1987 at DuPont in Wilmington. His research interests center on the synthesis of novel polymeric compounds. 